\title{
Lung ultrasound findings in a 64-year-old woman with COVID-19
}

\author{
Adam Thomas MD BHSc, Greg Haljan MD, Anish Mitra MD MPH
}

Cite as: CMAJ 2020 April 14;192:E399 doi: 10.1503/cmaj.200414; early-released March 31, 2020

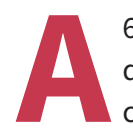

64-year-old female health care worker developed a sore throat and productive cough, which were followed by nausea, vomiting and exertional dyspnea over 1 week in March 2020. She had no travel history or obvious sick contacts, but had interacted with patients in an outpatient clinic. She presented to the hospital after outpatient viral polymerase chain reaction (PCR) testing was positive for severe acute respiratory syndrome coronavirus 2 (SARS-CoV-2), the virus responsible for coronavirus disease 2019 (COVID19). On presentation she was febrile $\left(38.7^{\circ} \mathrm{C}\right)$ and hypoxic (peripheral oxygen saturation $\left[\mathrm{SpO}_{2}\right] 88 \%$ on room air), but appeared comfortable. Bibasilar crackles were heard on auscultation, and her chest radiograph showed bilateral infiltrates. A lung ultrasound was obtained when the patient presented to hospital, 10 days after symptom onset, and showed multifocal B-lines, pleural thickening and subpleural consolidation (Figure 1). The patient was admitted to hospital, received supportive care, and her volume status was tightly regulated. She initially required $6 \mathrm{~L} / \mathrm{min} \mathrm{O}_{2}$ via nasal cannula, but 6 days after admission, her oxygen requirements increased and she was intubated. At the time of this submission, she is stable but remains on mechanical ventilation.

Our images are similar to the lung ultrasound findings of Peng and colleagues regarding COVID-19. ${ }^{1}$ However, similar findings were also described in influenza A (H1N1) pneumonia. ${ }^{2}$ In 1 small study of patients with clinical and epidemiologic features compatible with COVID-19, PCR testing was shown to identify only $71 \%$ of subsequently confirmed cases compared with computed tomography of the lung. ${ }^{3}$ We propose that lung ultrasonography may be useful in the workup of patients with suspected COVID-19, even though differentiating between different causes of viral pneumonia is not possible.

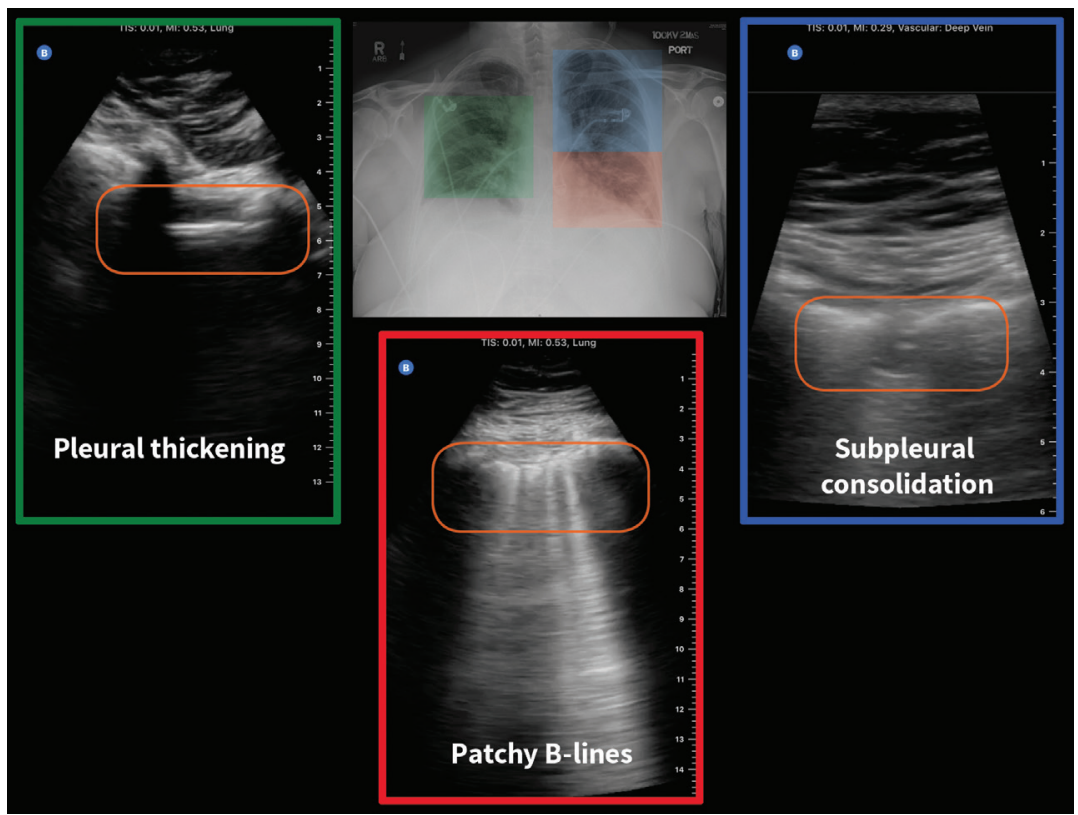

Figure 1: Lung ultrasound of a 64-year-old female health care worker with no travel history, on day 10 after symptom onset. The lung point-of-care utrasound shows pleural thickening (right lung: green box); subpleural consolidation, also known as "skip lesion" (left upper lung: blue box); and multifocal B-lines (left lung: red box). The chest radiograph shows bilateral infiltrates.

An excellent primer by Dr. Daniel Lichtenstein describes an approach to lung ultrasonography and discusses the findings and associated differential diagnoses in more detail. ${ }^{4}$

\section{References}

1. Peng QY, Wang XT, Zhang LN; Chinese Critical Care Ultrasound Study Group (CCUSG). Findings of lung ultrasonography of novel corona virus pneumonia during the 2019-2020 epidemic. Intensive Care Med 2020 Mar. 12 [Epub ahead of print]. doi: 10.1007/s00134-020-05996-6.

2. Testa A, Soldati G, Copetti R, et al. Early recognition of the 2009 pandemic influenza A (H1N1) pneumonia by chest ultrasound. Crit Care 2012;16:R30.

3. Fang Y, Zhang H, Xie J, et al. Sensitivity of chest CT for COVID-19: comparison to RTPCR. Radiology 2020 Feb. 19 [Epub ahead of print]. doi: 10.1148/radiol.2020200432.

4. Lichtenstein DA. Lung ultrasound in the critically ill. Ann Intensive Care 2014;4:1.
Competing interests: None declared.

This article has been peer reviewed.

The authors have obtained patient consent.
Affiliations: Department of Critical Care Medicine (Thomas, Haljan, Mitra), University of British Columbia, Vancouver, BC; Island Health and Victoria General Emergency Department (Thomas), Victoria, BC; Depart- ment of Critical Care (Haljan, Mitra), Fraser Health, Surrey, BC

Correspondence to: Anish Mitra, anish.mitra@fraserhealth.ca 